\title{
Various aspects of Contrast-enhanced Ultrasonography in splenic lesions - a pictorial essay
}

\author{
Elena Simona Ioanițescu ${ }^{1,2}$, Ionel Copaci ${ }^{1,2}$, Eugenia Mîndruț ${ }^{1}$, Otilia Motoi ${ }^{1,2}$, Adriana \\ Mercan Stanciu ${ }^{1}$, Letiția Toma ${ }^{1,2}$, Elena Laura Iliescu ${ }^{1,2}$
}

${ }^{1}$ Fundeni Clinical Institute, Department of Internal Medicine, ${ }^{2 “ C}$ Carol Davila" University of Medicine and Pharmacy, Bucharest, Romania

\begin{abstract}
Spleen pathology is rare in comparison with other abdominal organs, but often its lesions occur in complex pathological contexts, with systemic involvement. Although the lesions could be discretely symptomatic, their evolutionary potential might be severe. Conventional B-mode and Doppler ultrasound are the first-line imaging methods in spleen assessment, but frequently they do not allow the characterization of focal splenic abnormalities. Contrast-enhanced ultrasound (CEUS) is fast, safe, easy to perform, non-irradiating and can be used in patients with renal failure. By highlighting splenic macro and micro-vascularization, CEUS significantly increases the detection rate and allows the characterization of vascular (infarction, ischemia, thrombosis) and traumatic lesions, with high diagnostic accuracy. Additionally, ectopic splenic tissue can be identified with high accuracy. The method improves both the detection and characterization of splenic nodules, but some limitations still remain, especially in the differential diagnosis between malignant and some benign lesions. This article aims to portray aspects of CEUS imaging in various splenic pathologies using clinical examples from our experience and to review the CEUS contribution in the diagnosis of splenic lesions.
\end{abstract}

Keywords: ultrasound; contrast enhanced ultrasound (CEUS); spleen

\section{Introduction}

Spleen pathology is rare compared to other abdominal organs, but, far from being the "silent and forgotten" organ of the abdomen, the spleen is affected by various systemic disorders [1].

Ultrasonography (US) is the first-line imaging method for evaluation of the splenic parenchyma. However, the focal splenic lesions (FSL) are frequently difficult to

Received 23.03.2020 Accepted 05.05.2020

Med Ultrason

2020, Vol. 22, No 3, 356-363

Corresponding author: Elena Simona Ioanițescu

Department of Internal Medicine,

Fundeni Clinical Institute, 258 Fundeni Street, 022328, Bucharest, Romania

Phone: +40 722494586

E-mail: simona.ioanitescu@gmail.com characterise only by B-mode and Doppler US. Contrastenhanced ultrasound (CEUS) offers several undeniable advantages, by being a fast, portable, non-irradiating method, without nephrotoxicity and relatively cheap when compared to computed tomography (CT) scan and magnetic resonance imaging (MRI).

The aim of this pictorial essay is to describe CEUS particularities in different splenic lesions, with illustrative examples from our experience.

\section{Examination technique}

CEUS should be performed only after complete Bmode and Doppler ultrasound examination, when baseline examination is inconclusive, in order to establish a definitive diagnosis [2,3]. In order to obtain the best image, it is recommended to use the highest frequency available probe, tailored to the depth of the targeted area 
[2]. Usually, a convex 3-5 MHz probe is used. In children or slim patients, a 7.5-12 MHz linear probe can be used.

As contrast agent (CA), SonoVue (Bracco, Italy) in a volume of 1-1.2 $\mathrm{ml}$ for convex probe and 2-2.4 $\mathrm{ml}$ for a linear probe is used in our department.

\section{Ectopic spleen tissue}

An accessory spleen (splenunculus) is usually located near the spleen and can be easily diagnosed by conventional US. Difficulties occur in ectopic spleen diagnosis, which can be easily confused with tumors or pathological adenopathies. On CEUS, splenosis shows vascular dynamics similar to that of the normal spleen [4]. Small nodules may also show homogeneous arterial enhancement. The CEUS diagnosis is based on the persistence of enhancement in the late-phase for more than 5 minutes. A particular situation we encountered was splenosis located in contact with liver capsule, mimicking a liver tu- mor. The three phases contrast dynamics both in liver and spleen and the enhancement more intense in spleen tissue could have supported misdiagnosis, but the correlation of CEUS data with patient's medical history and normal biological tests suggested splenosis (fig 1).

\section{Splenic infarction}

Splenic infarctions are some of the most common FSL, caused by different hematologic (sickle-cell anemia, thrombophilia, hematologic malignancies) or immunological disorders $[5,6]$. CEUS allows the diagnosis in the acute phase showing a non-enhancing area during the entire exploration and enables the correct assessment of its shape and extent with a very high diagnostic accuracy. The lesion is clearly visible during venous and late phase. One particular etiology we must keep in mind is endocarditis. Small infarctions due to septic emboli from infectious endocarditis can be accurately detected by CEUS
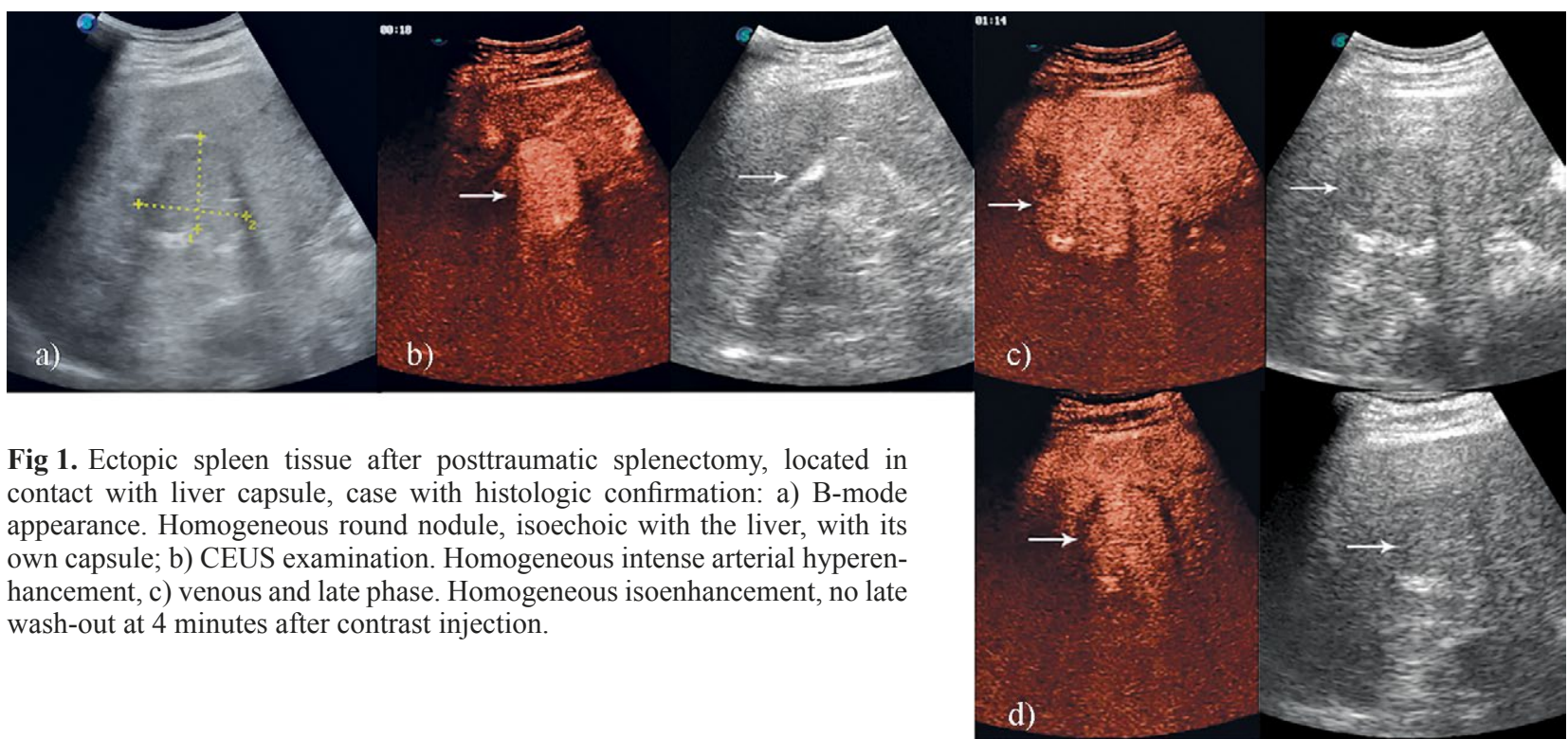

Fig 1. Ectopic spleen tissue after posttraumatic splenectomy, located in contact with liver capsule, case with histologic confirmation: a) B-mode appearance. Homogeneous round nodule, isoechoic with the liver, with its own capsule; b) CEUS examination. Homogeneous intense arterial hyperenhancement, c) venous and late phase. Homogeneous isoenhancement, no late wash-out at 4 minutes after contrast injection.

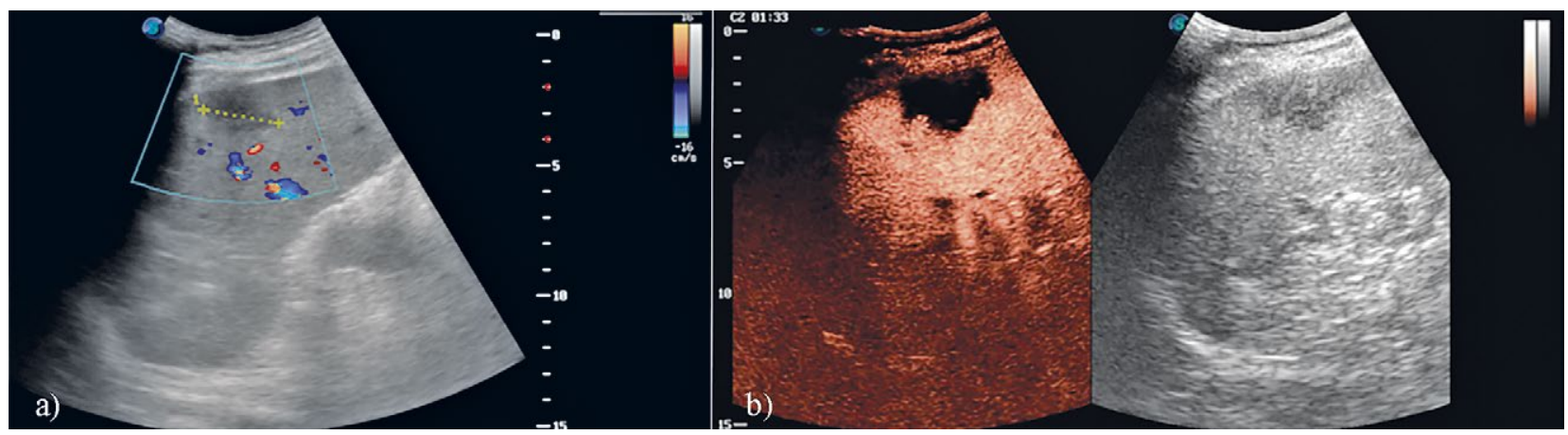

Fig 2. Splenic infarction in a 63 years old male, with recurrent fever, dyspnea and abdominal pain, diagnosed with endocarditis: a) hypoechoic area on B-mode examination, without Doppler signal inside; b) venous phase. On CEUS examination no contrast enhancement can be seen inside the infarction area. 
(fig 2) [7]. Splenic infarctions may also appear as complications of chemoembolization for hepatocellular carcinoma (HCC) [6]. Other successful applications of CEUS also used in our department are to evaluate the necrotic area and arterial permeability after therapeutic partial embolization of the splenic artery branch in cirrhotic patients (fig 3) or to confirm splenic vein permeability (fig 4).

\section{Splenic trauma}

Although contrast-enhanced CT remains the primeline investigation in politrauma patients, studies proved the utility of CEUS in the diagnosis, minimally invasive therapy, and follow-up of minor, blunt trauma, especially in children and young women [8-10]. Due to its arterial inhomogeneity and long-lasting contrast enhancement, the spleen is examined after the kidneys and liver, the lesions being clearly visible in the venous and late phase. The US appearance varies depending on the time between trauma and examination and lesions can be missed in the first 24 hours. CEUS significantly improves diagnosis showing hematomas and lacerations appearing as non-enhanced areas and contusions as ill-defined, hypoenhancing areas (fig 5) [3]. Sometimes, contrast extravasation as a sign of active bleeding can be seen within the splenic parenchyma or perisplenic hematomas [3].

\section{Splenic abscess}

Splenic abscesses are very rare and generally occur by hematologic dissemination from a primary infection site [11]. Bacterial abscesses are usually solitary, larger
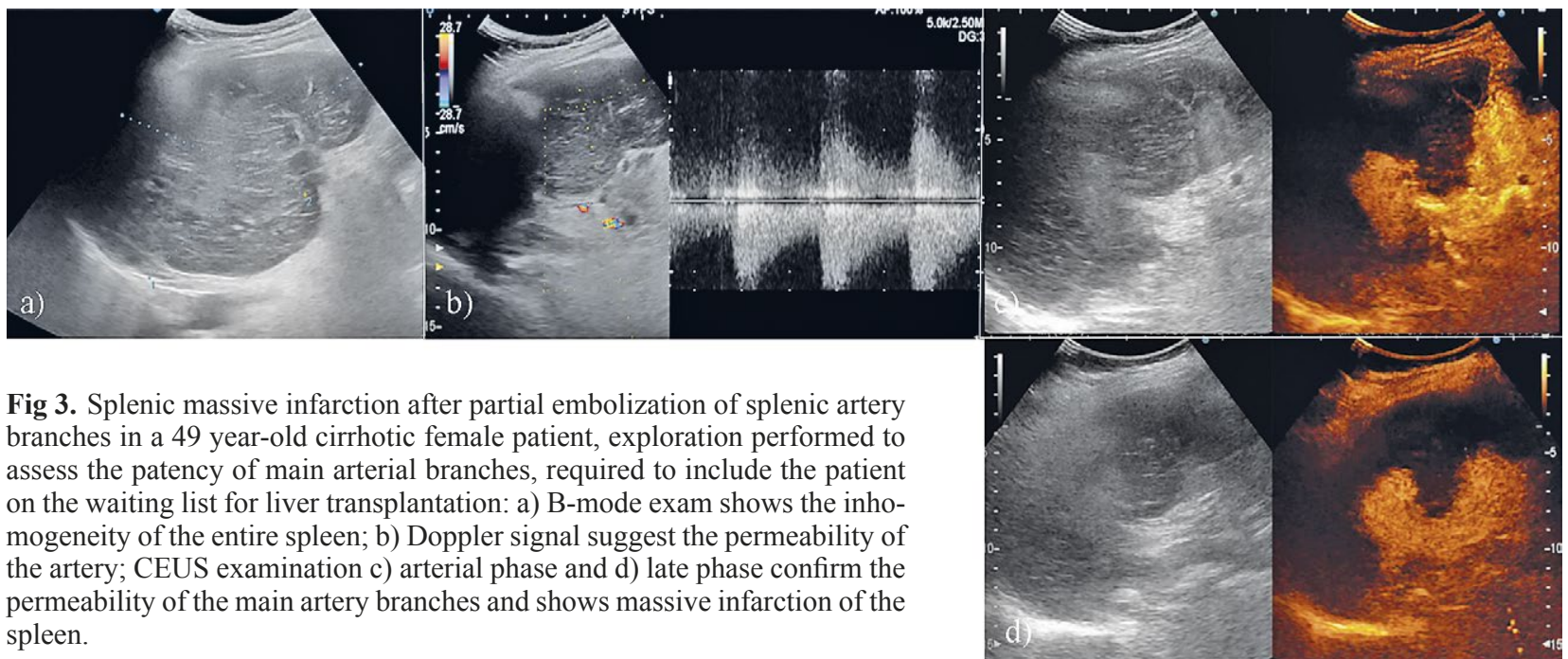

Fig 3. Splenic massive infarction after partial embolization of splenic artery branches in a 49 year-old cirrhotic female patient, exploration performed to assess the patency of main arterial branches, required to include the patient on the waiting list for liver transplantation: a) B-mode exam shows the inhomogeneity of the entire spleen; b) Doppler signal suggest the permeability of the artery; CEUS examination c) arterial phase and d) late phase confirm the permeability of the main artery branches and shows massive infarction of the spleen.
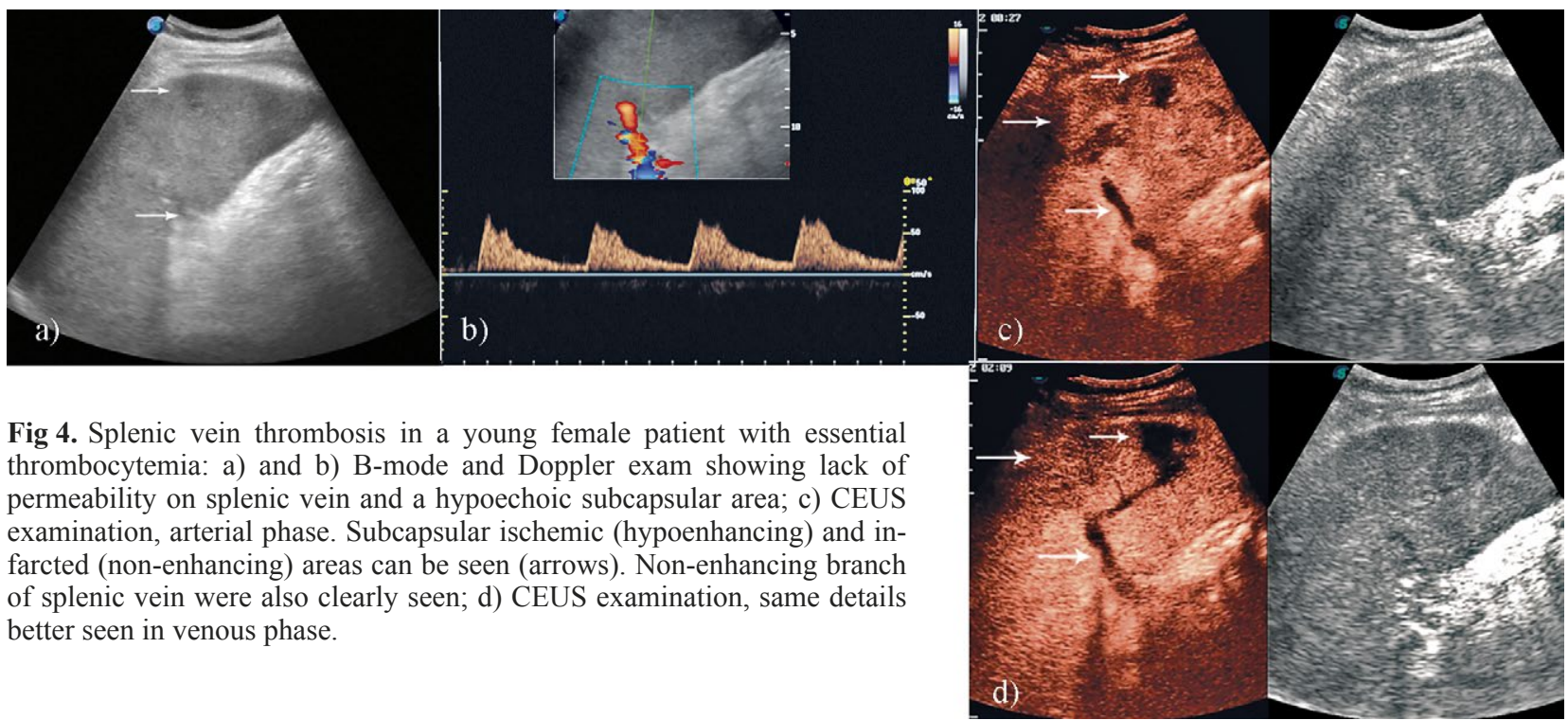

Fig 4. Splenic vein thrombosis in a young female patient with essential thrombocytemia: a) and b) B-mode and Doppler exam showing lack of permeability on splenic vein and a hypoechoic subcapsular area; c) CEUS examination, arterial phase. Subcapsular ischemic (hypoenhancing) and infarcted (non-enhancing) areas can be seen (arrows). Non-enhancing branch of splenic vein were also clearly seen; d) CEUS examination, same details better seen in venous phase. 


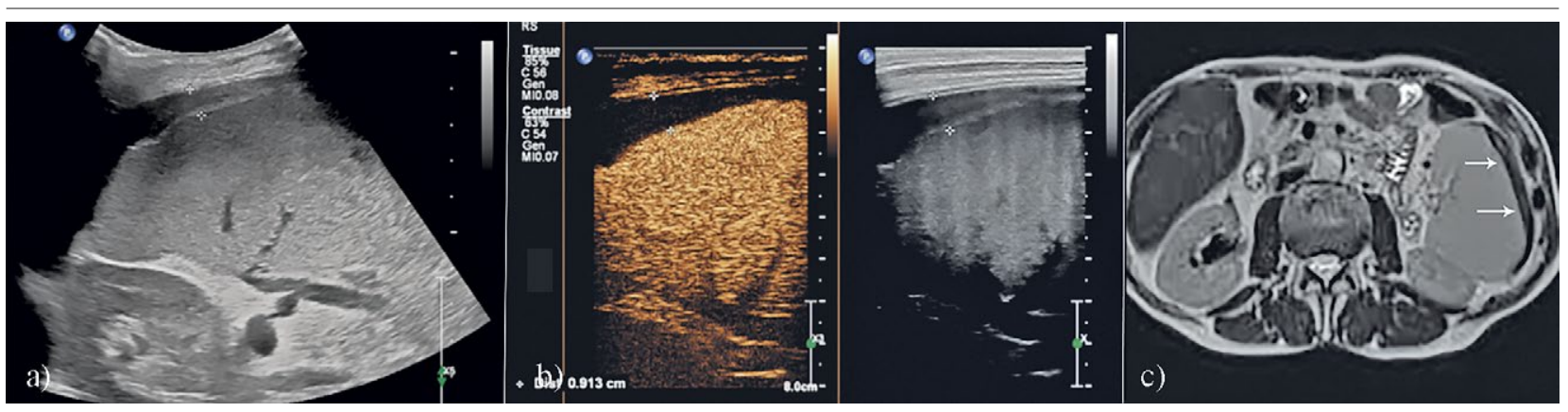

Fig 5. Subcapsular hematoma in a hepatocellular carcinoma patient who developed left quadrant abdominal pain 24 hours after chemoembolization: a) B-mode appearance. Heterogeneous area subcapsulary located; b) On CEUS examination no contrast enhancement of the subcapsular area can be seen; c) Computer-tomography examination confirms the subcapsular hematoma (arrows).

than $2 \mathrm{~cm}$, with B-mode and CEUS appearance similar to those from liver. Fungal abscesses are small, multiple, hypoechoic or target-like lesions and usually appear in immune compromised patients. On CEUS they appear non-enhancing or with a thin arterial hyperenhancing rim, that washes out in the venous and late phase.

\section{Splenic hemangiomas}

Splenic hemangiomas are the most common incidentally found benign splenic focal nodules (SFN) and their contrast enhancement is different from liver hemangiomas [6]. Typical nodular hyper-enhancement seen in liver hemangiomas is found in only $7 \%$ of splenic hemangiomas [12]. Capillary hemangioma appears as small hyperechoic round, well delineated SFN that enhances on CEUS as a normal spleen and becomes undetectable, without late wash out [4]. On CEUS, arterial enhancement of the cavernous hemangioma may be centripetal or diffuse, faster or slower, complete or incomplete due to fibrosis or thrombosis [6]. In the late phase no wash out is typical, but studies reported discrete late wash out in up $2 / 3$ of cases (fig 6 , fig 7) [12,13].

\section{Splenic hamartomas}

Splenic hamartomas are rare benign SFN originating from the red pulp, often less than $3 \mathrm{~cm}$. They are composed of normal spleen tissue and dilated splenic sinusoids [6]. On B-mode US they may appear as cystic lesions with septa or as hypoechoic inhomogeneous nodules. Doppler examination shows hypervascular lesions, some of them having a wheel distribution of vessels. CEUS examination shows an intense and homogeneous, centripetal or centrifugal arterial hyper-enhancement and moderate late wash out [6].

It must be outlined that the differential diagnosis between splenic hemangiomas, hamartomas or lymphangioma is often difficult to perform, frequently the final diagnosis suggesting a benign angiomatous lesion, as hemangioma or hamartoma [6].

\section{Splenic cysts}

Usually, cysts are easily diagnosed on US, no other investigations being necessary. CEUS is recommended only for complicated cysts if diagnosis on B-mode US is unclear. At CEUS they are non-enhancing during the en-

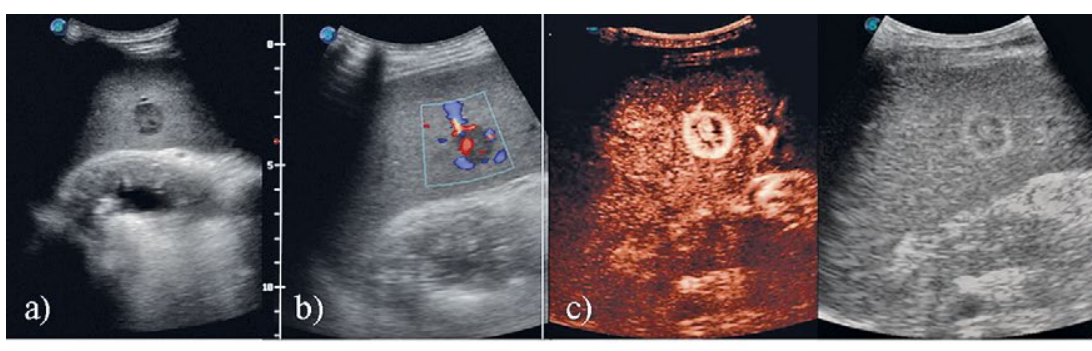

Fig 6. Small splenic hemangioma in a cirrhotic patient, incidental finding, a case with CT confirmation: a) B-mode examination - small hypoechoic round lesion; b) intense intralesional Doppler signal; c) CEUS exploration - rapid and intense arterial hyperenhancement; d) venous phase - complete homogeneous enhancement; e) late phase - discrete late wash-out.

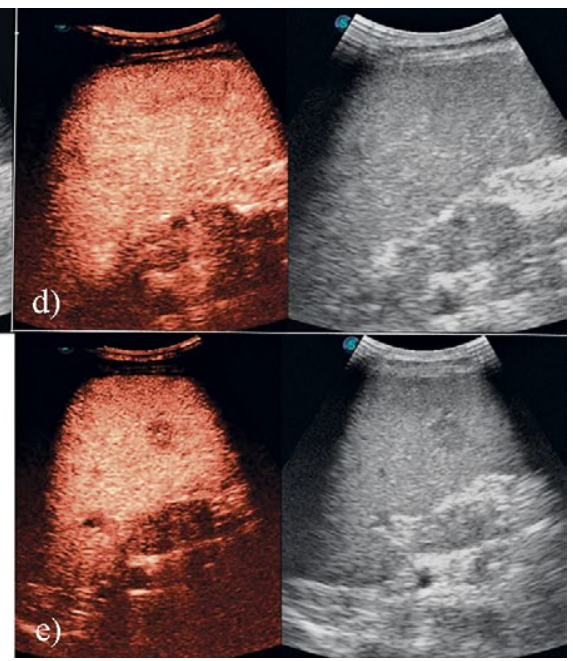



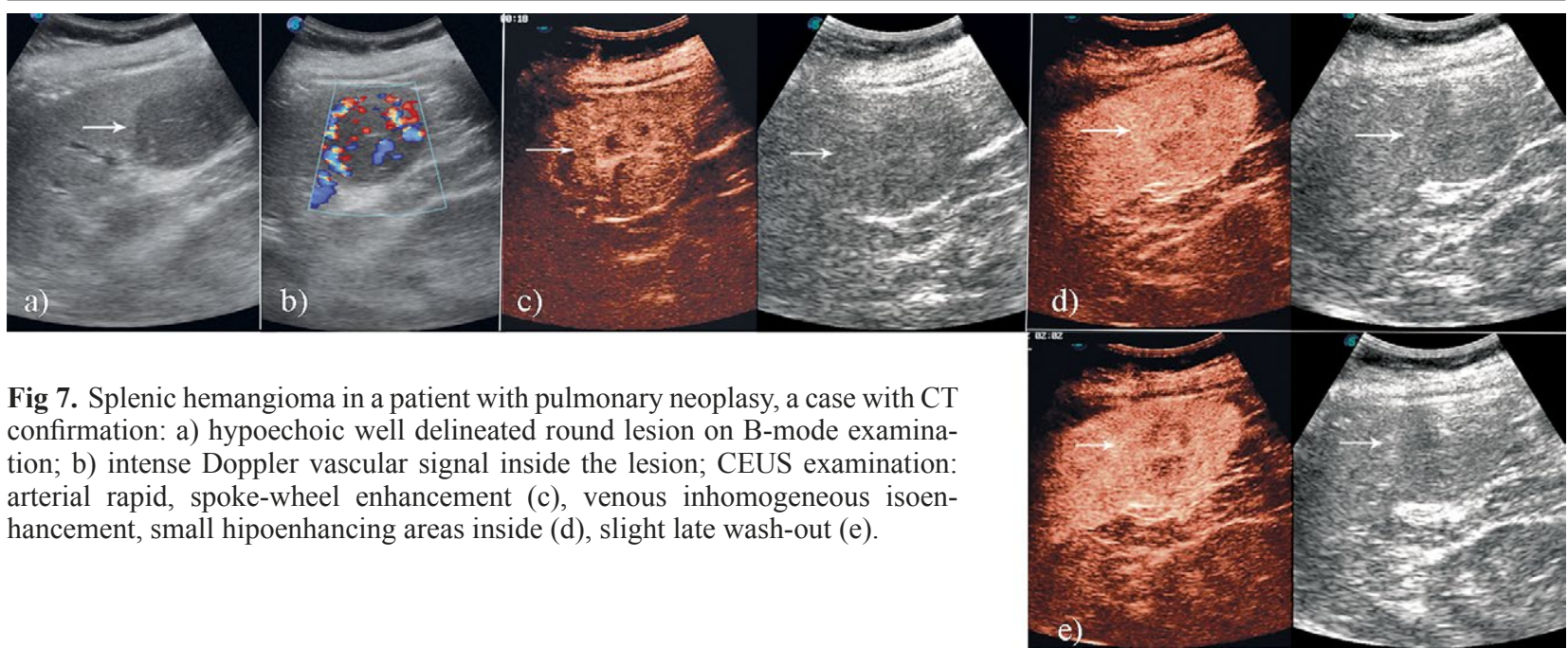

Fig 7. Splenic hemangioma in a patient with pulmonary neoplasy, a case with CT confirmation: a) hypoechoic well delineated round lesion on B-mode examination; b) intense Doppler vascular signal inside the lesion; CEUS examination: arterial rapid, spoke-wheel enhancement (c), venous inhomogeneous isoenhancement, small hipoenhancing areas inside (d), slight late wash-out (e).
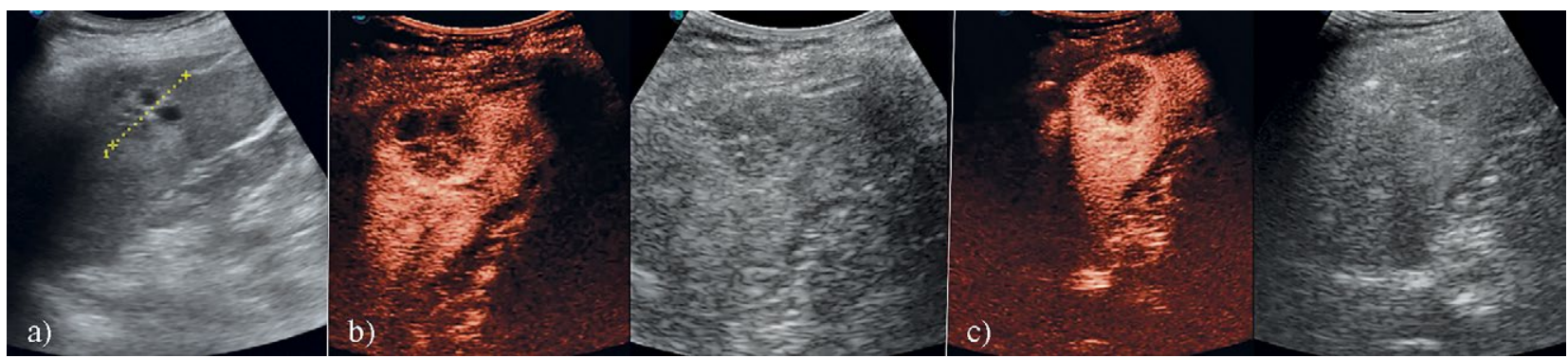

Fig 8. Two policystic lesions incidentally found in a 56 year old female, suspicion lymphangioma. First lesion with discretely dimensional progression at 1-year follow-up: a) B-mode examination - isoechoic, poor delineated lesion containing multiple small cysts; CEUS examination shows arterial peripheral rim hyperenhancement, slightly hypoenhancing septa, non-enhancing cysts (b) late phase - slight intralesional wash-out (c).

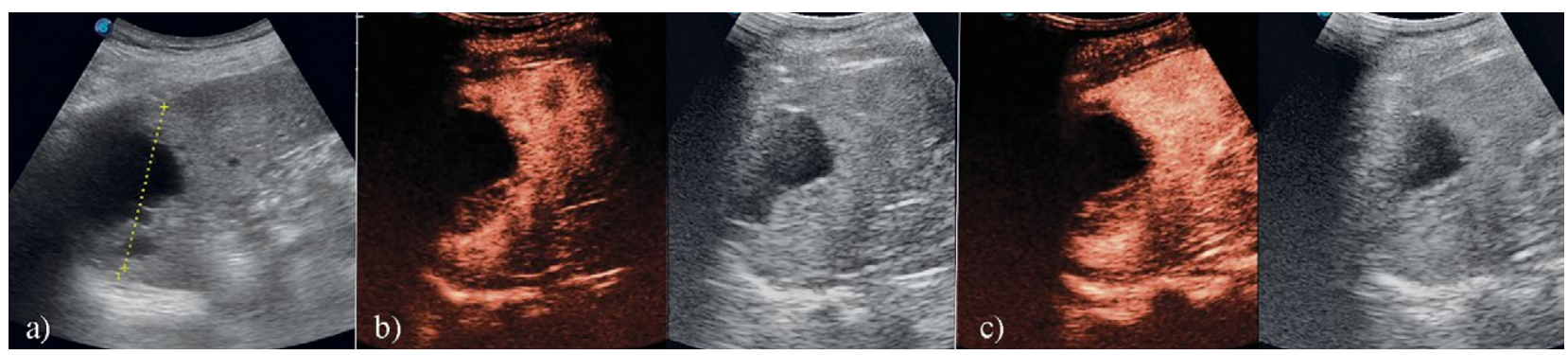

Fig 9. Same case as in figure 11. Second lesion with certain dimensional progression of the main cyst. CT scan also suggests lymphangioma. The patient refuses splenectomy at that moment. (a) B-mode appearance as isoechoic, poor delineated lesion containing multiple cysts, we note the diameter of the lesion and one cyst more than $5 \mathrm{~cm}$ diameter; (b) arterial peripheral rim hyperenhancement, slightly hypoenhancing septa, non-enhancing cysts (c) discrete intralesional wash-out.

tire exploration. But CEUS alone might be inconclusive in differential diagnosis between small cysts and fungal abscesses, granulomas or necrotic metastases and complementary investigations are necessary.

\section{Lymphangioma}

Lymphangioma is a rare congenital malformation of the lymphatic duct, usually associated with capillary, cavernous or cystic splenic lymphangiomas. They are usually located in the subcapsular area. The cystic form is the most common. B-mode US usually shows a solitary lesion containing multiple hypoechoic cysts of various sizes, with internal debris or septa [6]. On Doppler exam, the walls and septa may show vascular signal. On CEUS, they present arterial enhancement at the capsule and septum level, with or without discrete late wash-out (fig 8, fig 9) [2]. 


\section{Splenic granulomatous lesions}

Splenic involvement is commonly seen in tuberculosis or sarcoidosis. Usually, on B-mode US, granulomas are multiple, small, hypoechoic, well delineated lesions. They may be associated with microcalcifications, especially tuberculosis granuloma. On CEUS, they are usually hypoenhancing in the arterial phase, less frequently isoenhancing, with rapid and pronounced wash out, which makes them difficult to differentiate from malignant nodules. Difficulties also arise in differential diagnosis between granulomas and miliary abscesses, since both types of lesions are found in immune compromised patients (fig 10).

\section{Lymphoma}

Lymphoma is the most common splenic malignancy [2], splenic involvement being found at diagnosis in up to $30 \%$ of patients, more frequently in non-Hodgkin's lymphoma. The US findings vary: homogeneous splenomegaly, miliary nodules, multiple FSL or unique, macronodular, "bulky" lesion. On CEUS, the lymphoma lesions have, in most cases, iso- or discrete arterial hypoenhancing, with rapid, pronounced, and progressive wash out, starting less than 1 minute after infusion (fig 11) $[12,14]$. Studies show that the wash out in malignant SFN appears faster and is more pronounced than in benign lesions.
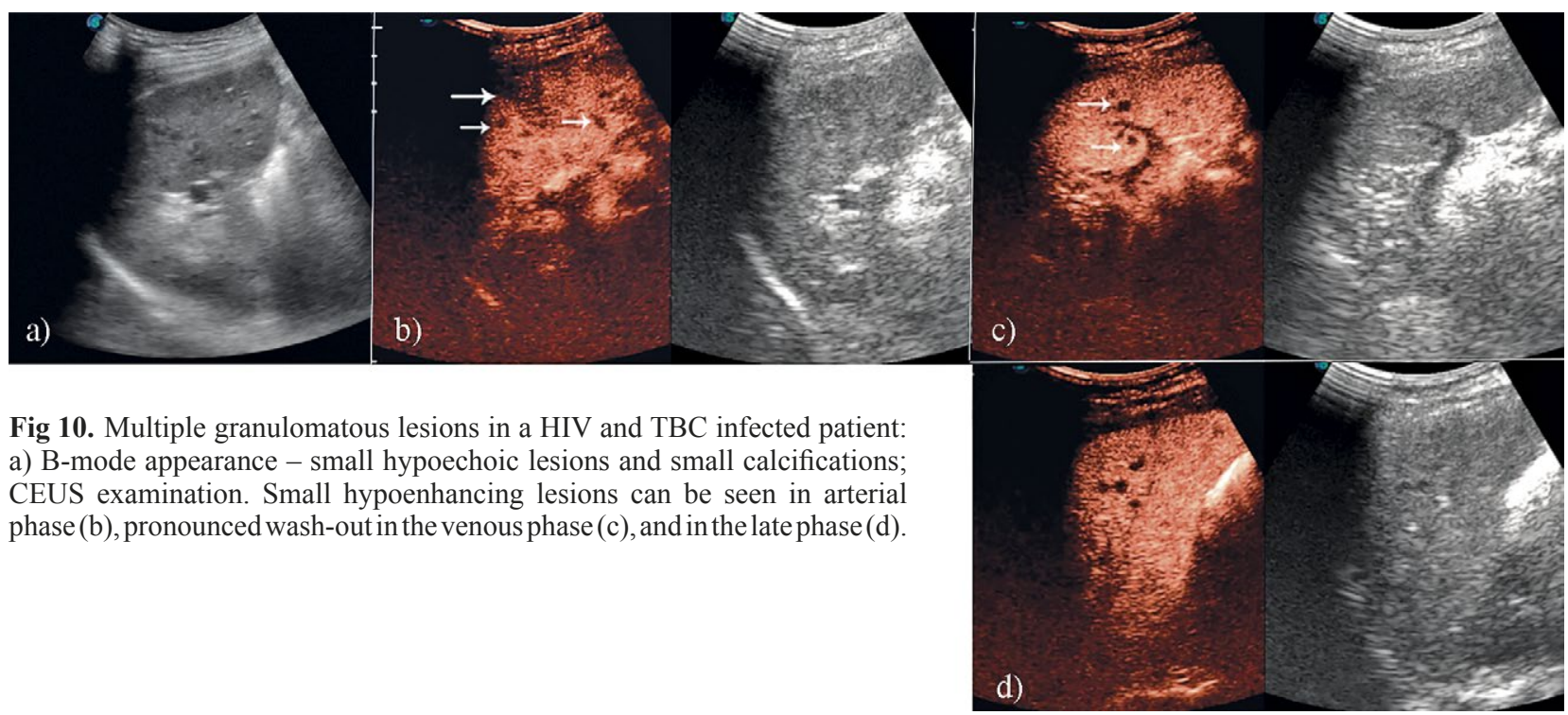

Fig 10. Multiple granulomatous lesions in a HIV and TBC infected patient: a) B-mode appearance - small hypoechoic lesions and small calcifications; CEUS examination. Small hypoenhancing lesions can be seen in arterial phase (b), pronounced wash-out in the venous phase (c), and in the late phase (d).
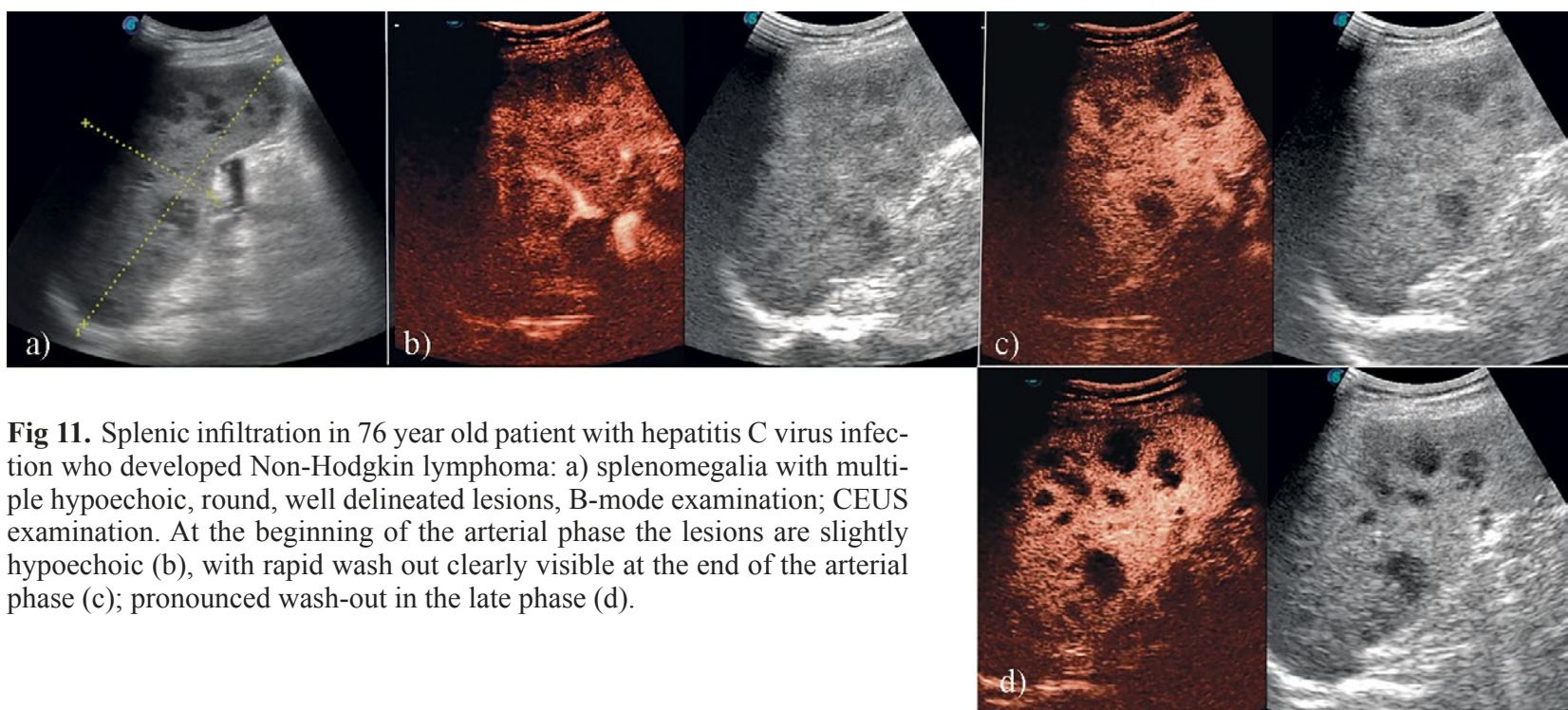

Fig 11. Splenic infiltration in 76 year old patient with hepatitis $C$ virus infection who developed Non-Hodgkin lymphoma: a) splenomegalia with multiple hypoechoic, round, well delineated lesions, B-mode examination; CEUS examination. At the beginning of the arterial phase the lesions are slightly hypoechoic (b), with rapid wash out clearly visible at the end of the arterial phase (c); pronounced wash-out in the late phase (d). 

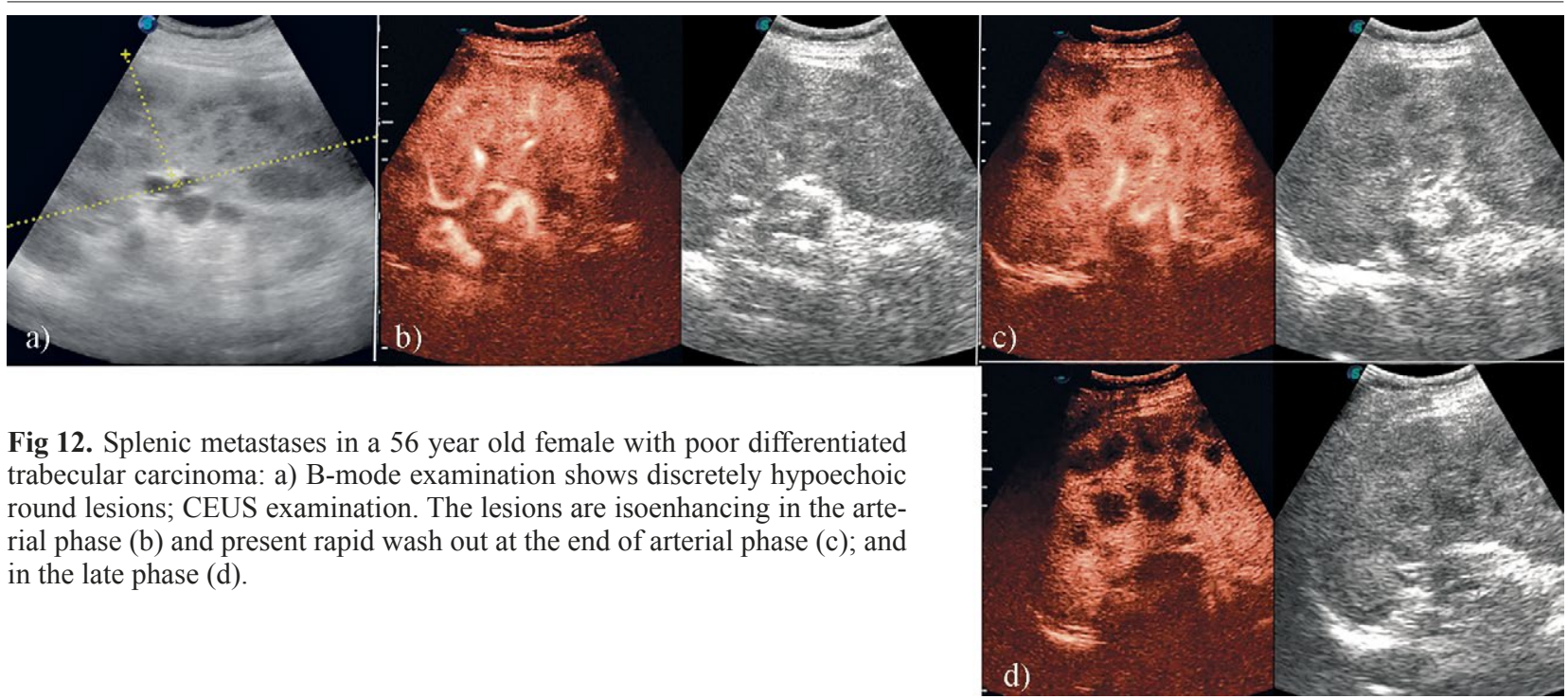

Fig 12. Splenic metastases in a 56 year old female with poor differentiated trabecular carcinoma: a) B-mode examination shows discretely hypoechoic round lesions; CEUS examination. The lesions are isoenhancing in the arterial phase (b) and present rapid wash out at the end of arterial phase (c); and in the late phase (d).

On the other hand, CEUS proved to be very useful for detection of lymphomatous infiltration. Studies showed $90 \%$ sensitivity and $100 \%$ specificity compared to CT for detection of lymphoma nodules [15]. Other studies showed CEUS sensitivity higher than CE-CT or FDG PET in detecting splenic lymphoma lesions in patients with Hodgkin's lymphoma [16].

\section{Splenic metastases}

Splenic metastases are very rare and occur late in the evolution of the disease. Most common, splenic metastasis occur from breast cancer, lung cancer, colorectal cancer, ovarian cancer, and malignant melanoma [17]. On CEUS, they usually present arterial enhancement similar to liver metastases, and rapid and pronounced wash out (fig 12).

Studies proved that CEUS increases the detection rate of splenic metastases by up to $40 \%$ compared to US [18]. CEUS is also useful in post-treatment follow-up after chemotherapy, avoiding multiple irradiations.

In conclusion, CEUS proves to be a very useful diagnostic method in daily practice, significantly increasing the detection rate of FSL. The method allows ectopic splenic tissue diagnosis with high accuracy. In case of infarction and traumatic lesions, CEUS allows a correct assessment of their extension and is very useful in the follow-up of those lesions conservatively managed. Regarding the characterization of SFN, CEUS still has a limited role, allowing the selection of splenic nodules with certain benign features, for which only serial imaging is recommended for follow-up. We strongly believe that current use of CEUS in clinical practice paralleling with $\mathrm{CT}$ and MRI increases the diagnostic accuracy of splenic lesions and significantly shortens the time to diagnosis.

\section{Conflict of interest: none}

\section{References}

1. Görg C. The forgotten organ: Contrast enhanced sonography of the spleen. Eur J Radiol 2007;64:189-201.

2. Zavariz JD, Konstantatou E, Deganello A, et al. Common and uncommon features of focal splenic lesions on contrast-enhanced ultrasound: a pictorial review. Radiol Bras 2017;50:395-404.

3. Catalano O, Sandomenico F, Matarazzo I, Siani A. ContrastEnhanced Sonography of the Spleen. AJR Am J Roentgenol 2005; 184:1150-1156.

4. Sidhu P, Cantisani V, Dietrich CF, et al. The EFSUMB Guidelines and Recommendations for the Clinical Practice of Contrast-Enhanced Ultrasound (CEUS) in Non-Hepatic Applications: Update 2017 (Long Version). Ultraschall Med 2018;39:e2-e44.

5. Hopkins A, Cokkinos DD, Antypa EG, Piperopoulos PN. Contrast enhanced sonographic study of the spleen. Hell J Radiol 2017;2:49-65.

6. Caremani M, Occhini U, Caremani A, et al. Focal splenic lesions: US findings. J Ultrasound 2013;16:65-4.

7. Menozzi G, Maccabruni V, Gabbi E, Magnani G, Garlassi E. Evaluation with contrast ultrasound of the prevalence of splenic infarction in left-sided infective endocarditis. J Ultrasound 2014;18:223-227.

8. Miele V, Piccolo CL, Galluzzo M, Ianiello S, Sessa B, Trinci M. Contrast-enhanced ultrasound (CEUS) in blunt abdominal trauma. Br J Radiol 2016;89:20150823.

9. Tagliati C, Argalia G, Giuseppetti GM. Contrast-enhanced ultrasound performance in predicting blunt splenic injuries requiring only observation and monitoring. Med Ultrason 2019;21:16-21. 
10. Tang J, Zhang H, Lv F, et al. Percutaneous Injection Therapy for Blunt Splenic Trauma Guided by Contrast-Enhanced Ultrasonography. J Ultrasound Med 2008;27:925-932.

11. Ferraioli G, Brunetti E, Gulizia R, Mariani G, Marone P, Filice C. Management of splenic abscess: report on 16 cases from a single center. Int J Infect Dis 2009;13:524-530.

12. von Herbay A, Barreiros AP, Ignee A, et al. Contrast-enhanced ultrasonography with SonoVue: differentiation between benign and malignant lesions of the spleen. J Ultrasound Med 2009;28:421-434.

13. Taibbi A, Bartolotta TV, Matranga D, Midiri M, Lagalla R. Splenic hemangiomas. contrast-enhanced sonographic findings. J Ultrasound Med 2012;31:543-553.

14. Iliescu L, Mercan-Stanciu A, Ioanitescu ES, Toma L. Hepatitis C-Associated B-cell Non-Hodgkin Lymphoma: A Pictorial Review. Ultrasound Q 2018;34:156-166.
15. Tafuto S, Catalano O, Barba G, et al. Real-time contrastenhanced specific ultrasound in staging and follow-up of splenic lymphomas. Front Biosci 2006;11:22242229.

16. Picardi M, Soricelli A, Pane F, et al. Contrast-enhanced harmonic compound US of the spleen to increase staging accuracy in patients with Hodgkin lymphoma: a prospective study. Radiology 2009;251:574-582.

17. Compérat E, Bardier-Dupas A, Camparo P, Capron F, Charlotte F. Splenic metastases: clinicopathologic presentation, differential diagnosis, and pathogenesis. Arch Pathol Lab Med 2007;131:965-969.

18. Neesse A, Huth J, Kunsch S, et al. Contrast-enhanced ultrasound pattern of splenic metastases - a retrospective study in 32 patients. Ultraschall Med 2010;31:264269. 